\title{
ANÁLISE DE VARIÁVEIS AERÓBIAS E ANTROPOMETRICAS DE FUTEBOLISTAS PROFISSIONAIS, JUNIORES E JUVENIS
}

Ms. José Mário Campeiz

Preparador Físico do Mogi Mirim Futebol Clube

Dr. Paulo Roberto de Oliveira

Prof. Gustavo Bastos Moreno Maia.

Faculdade de Educação Física/UNICAMP

\section{Resumo}

O consumo máximo de oxigênio é a variável mais estudada no futebol. Vários estudos demonstram através de mensurações diretas e indiretas constituir-se uma variável indispensável para tornar o atleta apto ao desempenho de alto nível, bem como para recuperar dos esforços curtos e intensos característicos do jogo. O presente estudo objetivou avaliar o consumo máximo de oxigênio $\left(\mathrm{VO}_{2} \max \right)$ através do protocolo de Leger e Lambert (1982) e, as variáveis antropométricas, massa corporal magra (MCM), percentual de gordura corporal $(\% \mathrm{G})$. A amostra deste estudo foi composta por um grupo de (54) cinqüenta e quatro futebolistas do sexo masculino, divididos em 3 grupos: profissionais $(\mathrm{G} 1) \mathrm{n}=21$, idade média de $24,0 \pm 3,3$ anos, peso inicial 77,4 \pm $6,8 \mathrm{~kg}$ e estatura de $179,3 \pm 6,6 \mathrm{~cm}$; juniores (G2) $\mathrm{n}=18$, idade média de 17,8 $\pm 0,8$ anos, peso médio inicial 70,1 $\pm 4,8 \mathrm{~kg}$ e estatura de $176,6 \pm 4,4 \mathrm{~cm}$; juvenis (G3) $\mathrm{n}=15$, idade média de $15,9 \pm 0,8$ anos, peso médio inicial $67,1 \pm 6,0 \mathrm{~kg}$ e estatura média de $175 \pm 4,4 \mathrm{~cm}$., avaliados no inicio do período preparatório do campeonato da Federação Paulista de Futebol (FPF), serie A1. Concluiu-se que a variável MCM apresentou diferenças estatisticamente significantes entre o G1 $(69,09 \pm 5,42 \mathrm{~kg})$ e G2 $(62,98 \pm$ $4,25 \mathrm{~kg})$; entre o G1 $(69,09 \pm 5,42 \mathrm{~kg})$ e G3 $(56,92 \pm 4,66 \mathrm{~kg})$ e entre o $\mathrm{G} 2(62,98 \pm$ $4,25 \mathrm{~kg}) \mathrm{e}$ G3 $(56,92 \pm 4,66 \mathrm{~kg})(\mathrm{p}<0,05)$. As variáveis $\mathrm{VO}_{2} \max \mathrm{e} \% \mathrm{G}$ não apresentaram diferenças estatisticamente significante entre os grupos estudados $(\mathrm{p}<0,05)$.

Palavras-Chave: Futebol; Consumo máximo de oxigênio; Composição corporal; Potência aeróbia.

\section{Introdução}

$\mathrm{O}$ consumo máximo de oxigênio $\left(\mathrm{VO}_{2}\right.$ max.) é a variável fisiológica que melhor descreve a capacidade funcional dos sistemas cardiovascular e respiratório. É aceito como o índice que representa a capacidade máxima de integração do organismo em captar, transportar e utilizar o oxigênio para os processos aeróbios de produção de energia durante a contração muscular (DENADAI, 1999). Pode ser expresso em termos absolutos (1/min) ou relativo à superfície corpórea ( $\mathrm{ml} / \mathrm{kg} / \mathrm{min})$. 
No futebol, é uma das variáveis fisiológicas mais estudadas e os resultados de diversos estudos reforçam a sua importância, evidenciando que o estímulo do sistema cardiorrespiratório nos treinamentos tem merecido atenção prioritária quando comparado com o estímulo neuromuscular.

Durante as partidas os futebolistas realizam esforços curtos, intensos e decisivos caracterizados predominantemente por anaeróbios aláticos com uma concentração lática entre 4 a $9 \mathrm{mMol}$, sendo o metabolismo aeróbio requerido principalmente nos momentos de recuperação entre esses esforços.

Muitos estudos de mensuração e determinação do consumo máximo de oxigênio, com medições diretas e indiretas, foram feitos, podendo-se destacar Carzola e Fahri (1998), Silva et al. (1996, 1997, 1998a, 1998b, 1998c, 1999), Bangsbo (1994a), Bosco (1993), evidenciando a necessidade de valorização de uma preparação física bem desenvolvida, e apoiada em princípios bem fundamentados cientificamente. Esse parâmetro fisiológico é de grande importância para qualificar o nível de capacidade funcional dos futebolistas e para possibilitar a identificação do sistema energético mais adequado à produção e ressíntese da energia.

No presente estudo optou-se para avaliar e comparar a potência aeróbia $\left(\mathrm{VO}_{2}\right.$ máximo) de futebolistas profissionais, juniores e juvenis, através do multistage fitness test, proposto inicialmente por Léger e Lambert (1982), e que foi validado por Duarte e Duarte (2001), para adultos jovens com o intuito de estimar a condição cardiorrespiratória.

\section{Breve Revisão Bibliográfica}

$\mathrm{Na}$ literatura verifica-se, que o padrão de $\mathrm{VO}_{2} \max$ em futebolistas é de aproximadamente 55-60 ml/kg/min (AOKI, 2002). O mesmo autor, citando Agnevik (1979) encontrou nos futebolistas da seleção sueca, resultados de $56,5 \mathrm{ml} / \mathrm{kg} / \mathrm{min}$. Willians et al. (1973) apud Aoki, (2002) encontrou 57,8 ml/kg/min quando analisou futebolista da equipe do Aberdeen F. C. Apor (1988) citado por Aoki (2002), encontrou em futebolistas, primeiro, segundo, terceiro e quarto colocados do campeonato húngaro, valores de $66,6,64,3,63,3$ e $58,1 \mathrm{ml} / \mathrm{kg} / \mathrm{min}$, respectivamente. 
Rhodes et al. (1986) apud Aoki (2002), nos estudos com futebolistas da seleção canadense encontrou valores de $58,7 \mathrm{ml} / \mathrm{kg} / \mathrm{min}$. Diferentes valores encontraram Faina et al (1988) em estudos com futebolistas italianos amadores, profissionais e da seleção italiana, com os resultados de 64,1, 58,9 e 63,2 ml/ kg/min, respectivamente.

Para Silva et al. (1998 b), a dinâmica mais participativa dos atletas durante as partidas exige um valor mínimo de $60 \mathrm{ml} / \mathrm{kg} / \mathrm{min}$. Silva et al. (1998a), compararam o desempenho de futebolistas brasileiros com os dados existentes na literatura especializada sobre futebol, demonstraram que os índices de limiar anaeróbio e $\mathrm{VO}_{2}$ máximo foram semelhantes e até mesmo superior a vários resultados de estudos publicados; por outro lado, ao se considerar as posições dos jogadores, afirmaram que não há um consenso definido sobre os níveis mais adequados dessa variável para os futebolistas.

Vários pesquisadores Carzola e Fahri (1998), Silva et al. (1999, 1998a, 1998b, 1998c, 1997, 1996), Bangsbo (1994a, 1994b), Bosco (1993) demonstram que o $\mathrm{VO}_{2}$ máximo é importante para a prática do futebol devido principalmente à longa duração do jogo. Portanto a produção de energia proveniente do sistema aeróbio parece suprir 80 a $90 \%$ da demanda energética durante uma partida de futebol, sendo recomendado aperfeiçoar e melhorar a eficiência do sistema oxidativo-aeróbio através do treinamento.

Losada apud Silva (1998a, 1998b), encontrou na seleção alemã (RFA), finalista e ganhadora da copa do Mundo de 1974, valores entre 66,0 e 68,0 e até mesmo 70,0 $\mathrm{ml} / \mathrm{kg} / \mathrm{min}$.

Entretanto, na opinião de Nowacki apud Silva et al. (1998b), valores acima de $70 \mathrm{ml} / \mathrm{kg} / \mathrm{min}$ ou níveis extremos de $85 \mathrm{ml} / \mathrm{kg} / \mathrm{min}$, tornam-se perigosos, pois podem comprometer a velocidade e a técnica dos jogadores; segundo o autor existe uma diminuição do fluxo metabólico alático e lático das fibras musculares, diminuindo a concentração e a atividade máxima de várias enzimas musculares envolvidas nos exercícios de alta intensidade; isto pode ser observado quando o treinamento aeróbio é realizado de forma volumosa.

Outros pesquisadores como Carzola e Farhi (1998), demonstraram que mais de $2 / 3$ da duração de uma partida são realizados com taxas superiores a $80 \%$ do $\mathrm{VO}_{2}$ 
máximo. Esses níveis seriam atingidos várias vezes numa partida (1 à 5 vezes, de acordo com a qualidade do jogo imposta pelo ou ao adversário); nos casos de igualdade técnica ou atlética, o jogador de alto nível se destaca por ter capacidade e potência aeróbia superiores. Enfim, alguns autores afirmam que o mais importante não é o valor absoluto do $\mathrm{VO}_{2}$ máximo e sim em que percentual do $\mathrm{VO}_{2}$ máximo o limiar de lactato é atingido.

Bangsbo et al. Apud Aoki (2002) afirmaram em seus estudos que os meiocampistas apresentam valores mais altos de $\mathrm{VO}_{2}$ máximo. Semelhantes observações foram feitas por Silva et al (1998a, 1998b) que afirmaram que duas posições merecem destaque pela solicitação física: os laterais e os meios campistas que são altamente exigidos durante o jogo pela elevada velocidade de corrida e alta porcentagem da fração do $\mathrm{VO}_{2}$ máximo no limiar anaeróbio.

Enfim, a variabilidade dos valores de $\mathrm{VO}_{2} \max (\mathrm{ml} / \mathrm{kg} / \mathrm{min})$ em futebolistas é grande, não somente pelas características individuais, mas em virtude dos diferentes modelos e metodologias utilizadas nos treinamentos e, também pelos diferentes tipos de ergômetros utilizados na avaliação, como bicicleta ou esteira. É sabido que valores de $\mathrm{VO}_{2}$ máximo obtidos nos protocolos realizados em bicicleta ergométrica são em média $5 \%$ a $20 \%$ inferiores àqueles observados em esteira (Hermansen e Saltin, apud Silva, $1998^{\mathrm{b}}$ ), devendo-se relativizar as conclusões dos estudos comparativos realizados com diferentes ergômetros e diferentes protocolos.

\section{Procedimentos Metodológicos}

Amostra

A amostra deste estudo foi composta por um grupo de 54 (cinqüenta e quatro) futebolistas do sexo masculino, divididos em 3 grupos: profissionais $(\mathrm{G} 1) \mathrm{n}=21$, idade média de $24,0 \pm 3,3$ anos, peso inicial $77,4 \pm 6,8 \mathrm{~kg}$ e estatura de $179,3 \pm 6,6 \mathrm{~cm}$; juniores (G2) $\mathrm{n}=18$, idade média de 17,8 $\pm 0,8$ anos, peso inicial 70,1 $\pm 4,8 \mathrm{~kg}$ e estatura de $176,6 \pm 4,4 \mathrm{~cm}$; juvenis (G3) $\mathrm{n}=15$, idade média de $15,9 \pm 0,8$ ano, peso médio inicial $67,1 \pm 6,0 \mathrm{~kg}$ e estatura média de $175 \pm 4,4 \mathrm{~cm}$, todos participantes da série A1 do Campeonato Estadual da Federação Paulista de Futebol (FPF). 


\subsection{Testes de controle}

Para a realização deste estudo, utilizou-se o teste proposto por Leger et al. (1982), com os resultados expressos pelo consumo máximo de oxigênio $\left(\mathrm{VO}_{2}\right.$ máximo); a avaliação da composição corporal foi feita através do protocolo de Faulkner (1968), com os resultados expressos pelo percentual de gordura $(\% \mathrm{G})$ e da massa magra

(MCM). As aplicações dos testes ocorreram no início do período preparatório de treinamento.

\subsubsection{Multistage fitness test}

Teste cujo protocolo consiste na execução de repetidas corridas de $20 \mathrm{~m}$ separadas por períodos de recuperação, onde a velocidade de deslocamento aumenta progressivamente, sendo indicada por sinais sonoros.

\subsubsection{Antropometria}

A avaliação antropometrica consistiu na mensuração de: peso corporal através de uma balança da marca Filizola, modelo 31, calibrada com precisão aproximada de $0,5 \mathrm{~kg}$; da estatura corporal através de um estadiômetro fixo na mesma balança com escala de 0,5 cm; dobras cutâneas mensuradas com um compasso (adipômetro) da marca Lange, incluindo as dobras cutâneas biceptal, triceptal, subescapular, axilar, suprailíaca, abdominal, coxa e panturrilha; estas dobras foram submetidas ao somatório para posterior análise do percentual de gordura e da massa magra, de todos os futebolistas participantes do estudo; também foi mensurada a circunferência de braço, coxa e panturrilha. Utilizou-se o protocolo de Faulkner (1968), para o cálculo do percentual de gordura e da massa magra.

\subsection{Análise Estatística}

Os dados foram analisados estatisticamente (SPSS 8.0) por meio da avaliação descritiva que compreendeu medidas de tendência central e dispersão e análise de variância ANOVA ONE WAY para medidas independentes, complementada com o 
teste de Scheffe's post hoc na tentativa detectar possíveis diferenças das médias dos grupos estudados. O nível de significância para rejeição da hipótese nula foi $p<0,05$.

\section{Apresentação e Discussão dos Resultados}

\subsection{Considerações sobre a alteração do indicador da capacidade aeróbia e} composição corporal estudadas dentro dos diferentes grupos

Poucos são os estudos relacionados com as variáveis aeróbias utilizando o Multistage fitness test proposto por Léger et al. (1989), e também escassos são os estudos relacionados com as variáveis antropometrica de futebolistas brasileiros, em suas diferentes categorias. Entretanto vários autores como Carzola e Fahri (1998), Silva et al. (1999, 1998a, 1998b, 1998c, 1997, 1996), Bangsbo (1994a, 1994b), Bosco (1993) demonstraram a importância do conhecimento e aprimoramento dessas variáveis para a melhoria do desempenho durante o jogo, utilizando metodologias, ergômetros e protocolos diferentes.

Os resultados obtidos no presente estudo são demonstrados de forma descritiva em valores médios e respectivos desvios padrão.

No Quadro 1 são apresentados os valores médios, desvios padrão e significância $(\mathrm{p}<0,05)$ das variáveis estudadas nos diferentes grupos avaliados.

Quadro 1: Média, desvio padrão, significância estatística $(p<0,05)$ e análise de variância (Anova One Way) para medidas independentes complementadas com o teste de scheffé nas diferentes variáveis pesquisadas nos diferentes grupos

\begin{tabular}{c|cccc}
\hline & $\begin{array}{c}\text { Profissionais } \\
(\mathrm{G} 1)\end{array}$ & $\begin{array}{c}\text { Juniores } \\
(\mathrm{G} 2)\end{array}$ & $\begin{array}{c}\text { Juvenis } \\
(\mathrm{G} 3)\end{array}$ & $\mathrm{p}$ \\
\hline $\mathrm{VO}_{2} \mathrm{max}$. & $50,21 \pm 3,21$ & $49,58 \pm$ & $49,53 \pm 2,90$ & \\
$(\mathrm{ml} / \mathrm{min} / \mathrm{kg})$ & & 2,89 & & \\
$\mathrm{M} . \mathrm{C} . \mathrm{M}$ & $69,09 \pm$ & $62,98 \pm$ & $56,92 \pm 4,66$ & $0,001^{*}$ \\
$(\mathrm{~kg})$ & $5,42^{\mathrm{ab}}$ & $4,25^{\mathrm{c}}$ & & \\
Percentual de & $10,70 \pm 1,40$ & $10,15 \pm$ & $10,02 \pm 0,84$ & \\
Gordura (\%) & & 0,83 & & \\
\hline $\mathbf{a = 1} \mathbf{1} \quad \mathbf{b}=\mathbf{1 \neq 3}$ & $\mathbf{c}=\mathbf{2} \neq \mathbf{3}$ & & &
\end{tabular}




\subsubsection{Consumo máximo de oxigênio}

A análise do Quadro 1, permite afirmar que os atletas do grupo 1 (profissionais), obtiveram resultados superiores ao do grupo 2 (juniores) e grupo 3 (juvenis), mas que não foram estatisticamente significantes. Bremer e Davis (1991) apud Aoki (2002) em seus estudos também não encontraram diferenças na capacidade aeróbia entre amadores e profissionais.

$\mathrm{O}$ consumo máximo de oxigênio $\left(\mathrm{VO}_{2} \mathrm{max}\right)$, tido durante muito anos como limitador do rendimento em várias modalidades principalmente as de resistência, ainda assume no futebol um papel prioritário para a resistência aeróbia em detrimento dos aspectos neuromusculares, mesmo na evidência de resultados de diferentes estudos demonstrarem a velocidade como fator determinante para o desempenho na modalidade.

Em diversas modalidades, como no futebol pela sua complexidade e intermitência de ações motoras, o aumento da capacidade oxidativa está relacionado não apenas ao aumento do $\mathrm{VO}_{2}$ máximo, mas também a uma série de outros fatores como número e o tamanho das mitocôndrias, que pode ser obtido através da preparação física especial. Para isto é necessário segundo Verkoshansky (1996), uma correta distribuição das cargas de treinamentos, da sucessão interconexão das cargas de diferente orientação fisiológica, das sessões de treinamentos, dos conhecimentos do heterocronismo dos sistemas energéticos mobilizados, volume e intensidade dos exercícios, enfim do conhecimento dos aspectos quantitativos e qualitativos que envolvem o treinamento e a competição dos futebolistas (CAMPEIZ; OLIVEIRA, 1999).

Entretanto os resultados obtidos neste estudo foram inferiores aos obtidos por Ekblom (1986), Holmann apud Dufor (1989) quando afirmaram que para os futebolistas correrem eficientemente durante os 90 minutos de jogo, o $\mathrm{VO}_{2}$ máximo deve ter valor entre 65 e $67 \mathrm{ml} / \mathrm{kg} / \mathrm{min}$. 
Gráfico 1: valores de $\mathrm{VO}_{2}$ máximo $(\mathrm{ml} / \mathrm{kg} / \mathrm{min})$ dos diferentes grupos do presente estudo.

\section{$\mathrm{VO}_{2}$ Máximo}

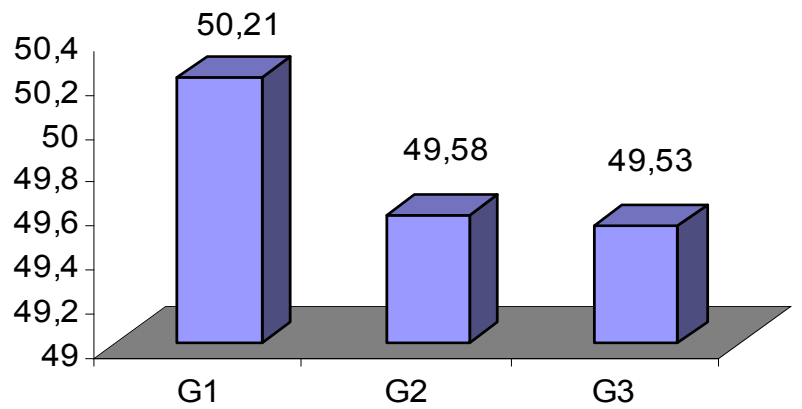

Os resultados do $\mathrm{VO}_{2}$ máximo obtidos no presente estudo de 50,21, 49,58 e $49,53 \mathrm{ml} / \mathrm{kg} / \mathrm{min}$., respectivamente para as categorias profissionais, juniores e juvenis (gráfico 1) também foram inferiores aos obtidos por Losada apud Silva (1998a, 1998b), Agnevik (1970) com futebolistas suecos (56,5 ml/kg/min), Apor (1998) apud Aoki (2002) com futebolistas húngaros (primeiro colocado: $66,6 \mathrm{ml} / \mathrm{kg} / \mathrm{min}$., segundo colocado: $64,3 \mathrm{ml} / \mathrm{kg} / \mathrm{min}$, terceiro colocado: $63,3 \mathrm{ml} / \mathrm{kg} / \mathrm{min}$, e quarto colocado: 58,1 $\mathrm{ml} / \mathrm{kg} / \mathrm{min}$ ), Rhodes et al. (1986), apud Aoki (2002) com futebolistas da seleção canadense $(58,7 \mathrm{ml} / \mathrm{kg} / \mathrm{min})$.

Berg et al. apud Silva (1998), encontrou valores entre 50,0 e 56,0 ml/kg/min em futebolistas de 12 anos de idade, resultados semelhantes aos dos juvenis (G3) e juniores (G2), e profissionais (G1) do presente estudo. Enquanto valores entre 50,0 e 52,0 $\mathrm{ml} / \mathrm{kg} / \mathrm{min}$ foram encontrados por Caru et al. apud Silva (1998c), em futebolista entre 14 e 18 anos de idade, Jones e Helmes apud Silva (1998c), encontraram valores entre 55,1 a $61,1 \mathrm{ml} / \mathrm{kg} / \mathrm{min}$. Apor apud Silva (1998c), encontrou valores médios de 73,9 $\mathrm{ml} / \mathrm{kg} / \mathrm{min}$ em 8 jogadores de 17 anos, pertencentes a clubes húngaros. Puga et al. (1993) citado por Aoki (2002) encontraram em futebolistas da primeira divisão portuguesa valores de $52,7 \mathrm{ml} / \mathrm{kg} / \mathrm{min}$. nos goleiros, $62,1 \mathrm{ml} / \mathrm{kg} / \mathrm{min}$. nos jogadores de defesas, $61,9 \mathrm{ml} / \mathrm{kg} / \mathrm{min}$. nos meio-campistas e $60,6 \mathrm{ml} / \mathrm{kg} / \mathrm{min}$. para os atacantes. Faina 
et al. (1988) apud Aoki (2002) pesquisaram futebolistas italianos amadores e encontraram resultados de $64,1 \mathrm{ml} / \mathrm{kg} / \mathrm{min}$, profissionais com $58,9 \mathrm{ml} / \mathrm{kg} / \mathrm{min}$ e 63,2 $\mathrm{ml} / \mathrm{kg} / \mathrm{min}$. para jogadores da seleção italiana. Todos esses resultados são superiores aos encontrados no presente estudo.

É importante salientar que as diferenças encontradas entre os valores de diferentes estudos provavelmente devam-se a utilização de diferentes tipos de protocolo, ergômetro ou metodologia utilizada para a avaliação e obtenção dos resultados, ou seja, o atleta pode reagir diferentemente de acordo com os protocolos, ou ainda, de acordo com a maior ou menor experiência no teste realizado.

Tabela 2: Comparação de valores de $\mathrm{VO}_{2}$ máximo $(\mathrm{ml} / \mathrm{kg} / \mathrm{min})$ do presente estudo com valores reportados de futebolistas de diferentes estudos.

\begin{tabular}{|c|c|c|c|}
\hline Futebolistas & $\begin{array}{c}\text { Valores } \\
(\mathrm{ml} / \mathrm{kg} / \mathrm{min})\end{array}$ & Categoria & Referências \\
\hline Mogi Mirim E.C. & $50,21 \pm 3,21$ & Profissional & Presente estudo \\
\hline Mogi Mirim E.C. & $49,58 \pm 2,89$ & Juniores & Presente estudo \\
\hline Mogi Mirim E.C. & $49,53 \pm 2,90$ & juvenis & Presente estudo \\
\hline Seleção Suécia & 56,6 & profissionais & Agnevik, (1979) \\
\hline Equipe Aberdeen F.C. & 57,8 & & Willians et al. (1973) \\
\hline Húngaros & 66,6 & profissionais & Apor (1888) \\
\hline Seleção Canadense & 58,7 & profissionais & Rhodes et al (1988) \\
\hline Italianos & 64,1 & Amadores & Faina et al. (1988) \\
\hline Italianos & 58,9 & Profissionais & Faina et al. (1988) \\
\hline Seleção Italiana & 63,2 & Profissionais & Faina et al. (1988) \\
\hline Seleção Alemã & $66-68$ & Profissionais & $\begin{array}{l}\text { Losada apud Silva (1998 } \\
\text { a,b) }\end{array}$ \\
\hline & $50-56$ & 12 anos & $\begin{array}{l}\text { Berg et al. apud Silva } \\
\text { (1998) }\end{array}$ \\
\hline & $50-52$ & 14-18 anos & Caru apud Silva (1998) \\
\hline $\begin{array}{l}\text { Portugueses da } 1^{\mathrm{a}} \text {. } \\
\text { divisão }\end{array}$ & $\begin{array}{l}52,7 \\
62,1 \\
61,9 \\
60,6\end{array}$ & $\begin{array}{l}\text { Profissionais } \\
\text { goleiros } \\
\text { defesas meio- } \\
\text { campo } \\
\text { atacantes }\end{array}$ & Faina et al. (1988) \\
\hline
\end{tabular}

\subsubsection{Massa corporal magra (MCM)}

A variável MCM apresentou diferença estatisticamente significante entre os três grupos de futebolistas analisados. Os profissionais (G1) com 69,09 $\pm 5,42 \mathrm{~kg}$, 
apresentaram resultados estatisticamente superiores aos juniores com $62,98 \pm 4,25(\mathrm{~kg})$ e juvenis com 56,92 \pm 4,66 kg. Os resultados dos juniores (G2) foram estatisticamente superiores quando comparados aos resultados dos juvenis (G3) (Gráfico 2).

Gráfico 2: valores de Massa Corporal Magra (kg) dos diferentes grupos do presente estudo.

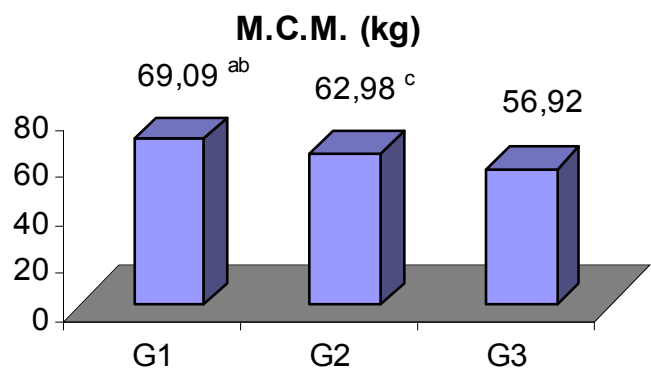

$$
a=1 \neq 2 \quad b=1 \neq 3 \quad c=2 \neq 3
$$

Sartori (2000), em estudos com futebolistas juniores relacionados à posição tática, e utilizando o mesmo tipo de protocolo do presente estudo, encontrou nos meiocampistas $61,02 \pm 4,30 \mathrm{~kg}$ e nos laterais 57,02 \pm 4,06 $\mathrm{kg}$ de M.C.M., resultados inferiores aos do presente estudo, quando comparados aos profissionais (G1). Entretanto, os resultados dos atacantes $63,46 \pm 4,17 \mathrm{~kg}$, goleiros $66,58 \pm 4,75 \mathrm{~kg}$ e zagueiros $67,62 \pm 5,91 \mathrm{~kg}$ foram superiores aos resultados do G2 e G3 do presente estudo.

Silva et al. (2000), em seus estudos concluíram que os jogadores de futebol juniores são mais altos e possui maior massa corporal magra $(43,0 \pm 5,6 \mathrm{~kg})$ em relação aos juvenis $(36,6 \pm 4,5 \mathrm{~kg})$. Afirma ainda, que a massa corporal magra é uma importante variável para prescrição do treinamento, pois está intimamente ligada à melhoria da potência e capacidade anaeróbia. 


\subsubsection{Percentual de gordura corporal (\% G)}

Os resultados apresentados no Quadro 1, demonstram uma homogeneidade entre os jogadores dos três grupos nesta variável. Como a variável antropométrica possibilita aos técnicos e cientistas do desporto avaliar, modificar e prescrever programas de treinamento (GREEN 1992), observa-se que o percentual de gordura constitui-se em um fator muito importante para o desempenho atlético Tricoli et al. apud Arruda e Rinaldi (1999). Assim sendo, o futebol como modalidade que envolve a execução de saltos verticais e deslocamentos rápidos e repetidos, qualquer excesso de peso corporal sob a forma de gordura pode levar a um prejuízo no rendimento. Os atletas do presente estudo apresentaram níveis considerados como ideais conforme os estudos de Green, (1992), Bangsbo, (1994), Godik (1996), Silva et al. (1999).

A variável \%G não apresentou diferença estatisticamente significante entre os diferentes grupos pesquisados (gráfico 3), evidenciando que desde as categorias juvenis até os profissionais as orientações dos conteúdos do treinamento e da nutrição desportiva parecem estar em acordo com o padrão corporal referenciado na literatura internacional.

Gráfico 3: Valores de percentual de gordura corporal (\%) dos diferentes grupos do presente estudo.

\section{$\%$ Gordura}

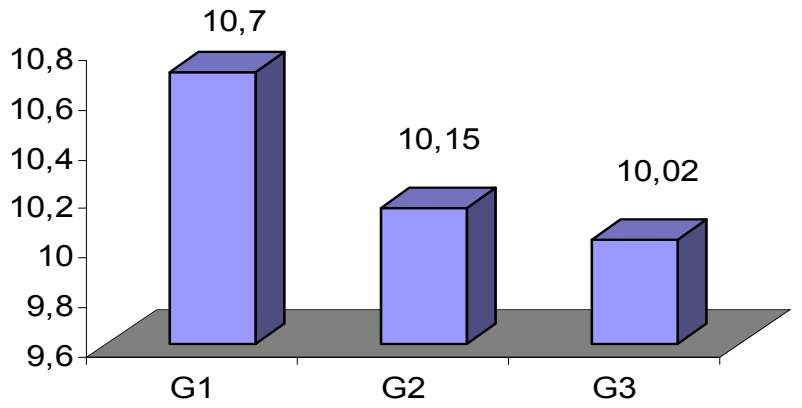


Os resultados encontrados neste estudo se mostraram inferiores aos resultados obtidos por Campeiz (2001), que analisou futebolistas profissionais submetidos a quatro avaliações em diferentes momentos de um macrociclo de treinamento. $\mathrm{O}$ autor utilizou o mesmo tipo de protocolo e encontrou no inicio da preparação (momento 1), media de $11,30 \pm 1,64 \%$. Também foram inferiores aos valores apresentados por Rienzi et al. apud Reilly et al. (2000), de futebolistas de nível internacional, participantes da copa América de 1995, que apresentaram resultados de 11\% de gordura corporal. Também inferiores aos dados encontrados por Berver e Davis (1992) apud Godik (1996), em 15 futebolistas profissionais da liga inglesa que apresentaram resultados de 11,0 $\pm 3,1 \%$, aos de Santos (1999), que utilizou o protocolo de Durnin e Womersley (1974) calculados pela formula de Siri (1961) e, finalmente, inferiores aos valores obtidos com futebolistas portugueses pertencentes à primeira divisão 11,4 \pm $2,6 \%$, segunda divisão $12,6 \pm 2,7 \%$, e quarta divisão $11,6 \pm 2,4 \%$, analisados na fase pré-competitiva. Há que se considerar a dificuldade de se comparar diferentes estudos, pois muitas vezes se desconhece o protocolo utilizado pelos autores.

Por outro lado, Bunc, Heller, Procuazka (1992) apud Godik (1996), estudaram 15 jogadores da seleção nacional da Checoslováquia e encontraram percentual de gordura corporal de 8,1 $\pm 2,7 \%$; Tokmakidis (1992) apud Godik (1996), encontrou com jogadores gregos valores de $9,2 \pm 1,6 \%$. Os resultados encontrados pelos autores acima são inferiores aos encontrados no presente estudo. Possíveis diferenças entre tais resultados e o resultado da presente pesquisa podem decorrer dos protocolos utilizados para o calculo do percentual de gordura corporal.

Silva et al. (2000) utilizando o mesmo tipo de protocolo do presente estudo, obtiveram resultados de $11,4 \pm 1,3 \%$ futebolistas juvenis comparado ao valor médio de $11,9 \pm 1,5 \%$ para futebolistas juniores do presente estudo (Quadro 1).

O valore médio do \%G do presente estudo foi semelhante ao obtido no início de preparação de futebolistas juniores do Mogi Mirim Esporte Clube (10,28 \pm 0,94\%), que utilizou o mesmo tipo de protocolo do presente estudo (não publicados).

Sartori (2000), utilizando o mesmo protocolo do presente estudo, avaliou futebolistas juniores conforme as posições táticas, encontrou nos zagueiros resultados 
médios de 9,97 $\pm 0,52 \%$. Davis et al. (1992) analisaram futebolistas da primeira e segunda divisão da liga inglesa, no momento pré-competitivo, através do protocolo de Durin e Womersley (1974) e, encontrou valores médios de $11,0 \%$. Os valores do presente estudo $(\% \mathrm{G})$ foram semelhantes aos obtidos por Campeiz et al. (2003); Campeiz (2001) que utilizou o mesmo protocolo.

A comparação dos valores médios encontrados nos atletas de diferentes categorias estudados G1, G2 e G3 comparados com os valores médios apresentados pelos diferentes autores acima citados, possibilita perceber um menor percentual de gordura no presente estudo. Por outro lado, os valores médios de $\mathrm{VO}_{2}$ máximo $(\mathrm{ml} / \mathrm{kg} / \mathrm{min}$.) mostram-se inferiores aqueles apresentados na literatura, nas diferentes categorias estudadas.

È importante refletir que os maiores valores de $\mathrm{VO}_{2}$ máximo encontrado nas décadas passadas (70 e 80), provavelmente sejam decorrentes da maior ênfase dada ao desenvolvimento da potência e capacidade aeróbia.

Parece que a atual tendência remete para novos pressupostos do desempenho no futebol baseados na particular atenção à preparação física especial, resistência especial, força, velocidade. Assim provavelmente, o menor nível de $\mathrm{VO}_{2}$ máximo (ml/kg:min.) apresentado pelos futebolistas da atualidade podem ser compensado por ajustes funcionais determinantes do desempenho.

\section{Conclusões}

Com base nos resultados encontrados neste estudo, pode-se concluir que:

1 - Das variáveis estudadas apenas a M.C.M apresentou resultados diferentes (estatisticamente significantes) entre os grupos estudados,

2 - Os resultados estatisticamente superiores de massa magra no grupo G1 (profissionais) não contribuiu para a obtenção de maiores valores de $\mathrm{VO}_{2}$ máximo pelo protocolo de Leger e Lambert. 
3 - Sugerem-se, outros estudos que verifiquem a possível influência da massa magra sobre o desempenho em testes contínuos, mas, principalmente utilizando-se de protocolos intermitentes ou, progressivos, com mudanças de direção, incluindo aceleração e desaceleração, dados as características intermitentes, com mudanças de direção peculiares do futebol.

\begin{abstract}
The maximum oxygen consumption is the most studied variable in soccer. Several studies show, through direct and indirect measuring, this to constitute an indispensable variable to make the athlete capable of a high level performance, and to recover from the short and intense effort which are characteristic of the game. This study has the objective of evaluating the maximum oxygen $\left(\mathrm{VO}_{2} \max \right)$ consumption through Leger and Lambert (1982) protocol, as well as the anthropometric variables, lean body mass, body fat percentage $(\% \mathrm{G})$. The sample for this study was composed of a group of fiftyfour (54) male soccer players separated into 3 groups: (G1) professional players $-\mathrm{n}=21$ individuals, average age 24 years $\pm 3,3$ years, initial weight 77.4 kilograms $( \pm 6.8 \mathrm{~kg})$ and height of $179.3 \mathrm{~cm}( \pm 6.6)$; (G2) junior $-\mathrm{n}=18$ individuals, average age $17.8( \pm$ 0.8 years), initial weight $70.1( \pm 4.8 \mathrm{~kg})$ and height of $176.6( \pm 4.4 \mathrm{~cm})$; and $(\mathrm{G} 3)$ juvenile $-\mathrm{n}=15$ individuals, average age 15.9 years $( \pm 0.8$ years), initial weight $67.1 \mathrm{~kg}$ $( \pm 6.0 \mathrm{~kg})$ and average height $175 \mathrm{~cm}( \pm 4.4)$, appraised at the beginning of the preparatory stage of the Federação Paulista de Futebol - FPF (São Paulo Soccer Federation) series A1. The study arrived to the conclusion that the Lean Body Mass showed differences statistically significant between the G1 $=69.09( \pm 5,42 \mathrm{~kg})$ and $\mathrm{G} 2$ $=62.98( \pm 4.25 \mathrm{~kg})$; between G1 and G3 $=56.92( \pm 4.66 \mathrm{~kg})$ and between G2 and G3 $(\mathrm{p}<0.05)$. The variables $\mathrm{VO}_{2} \max$ and $\% \mathrm{G}$ did not present statistically significant differences among the groups that were studied $(\mathrm{p}<0.05)$.
\end{abstract}

Key- Words: Soccer; Maximum oxygen consumption; Body mass; Aerobic energy 


\section{Referências Bibliográficas}

AL-HAZZAA, K. S.; ALMUZAINI, S. A.; AL-REFAEE, M. A.; SULAIMAN, M. Y.; DAFTERDAR,A.; AL-GHAMEDI,K.N.; AL-KURAIJI, K. N. Aerobic and anaerobic power characteristics of saudi elite soccer players. J. Sports Med Phys Fitness, v. 41, p. 54-61, 2001.

ANANIAS, G. E. O.; KOKOBUM, E.; MOLINA, R.; SILVA, P. R. S.; CORDEIRO, J. R. Capacidade funcional, desempenho e solicitação metabólica em futebolistas profissionais durante situação real de jogo monitorado por análise cinematográfica. Revista Brasileira de Medicina do Desporto, v. 4, n. 3, p. 87 - 95, 1998.

AOKI, M. S. Fisiologia, treinamento e nutrição aplicados ao futebol. Jundiaí, SP: Fontoura, 2002.

ARRUDA, M.; OLIVEIRA,P. R.; TOLEDO, N.; GOLULART, L. F. Futebol: uma nova abordagem de preparação física e sua influência na dinâmica da alteração dos índices de força rápida e resistência de força em um macrociclo. Revista Treinamento Desportivo, v. 4, n. 1, p. 23-28, 1999.

BANGSBO, J. The physyology of soccer with special reference to intense intermittent exercise. Acta Physiologica Scandinavica, v. 151, suppl. 619, 1994.

Energy demands in competitive soccer. Journal of Sports Sciences, v 12, p. 5 , 12.1994 .

Entrenamiento de la condición física en el fútbol. Barcelona: Paidotribo, 1997.

BOSCO, C. Aspectos fisiológicos de la preparación fisica del futbolista. Barcelona: Paidotribo, 1993.

CAMPEIZ, J. M., A Caracterização do esforço físico realizado durante uma partida de futebol. Revista Uniclar, vol 1, n. 6, p. 90 - 104, 1997.

Futebol: estudo da alteração de diferentes variáveis anaeróbias e da composição corporal em atletas profissionais durante um macrociclo de treinamento. Dissertação mestrado. Universidade Estadual de Campinas, Faculdade de Educação Física. Campinas, SP: 2001. 
.; OLIVEIRA, P. R. Futebol: contribuição com o estudo do princípio da sucessão interconexão da diferente orientação funcional predominante em curto período de preparação. Revista Faculdades Claretianas de Batatais, Batatais. v.8, p. 104-114, 1999.

; CAMPEIZ, E. C. F. S. Variáveis antropométricas, área muscular da coxa e nível de força máxima dos músculos extensores do joelho: estudo em profissionais de diferentes posições táticas. Revista Movimento \& Percepção,Espírito Santo do Pinhal, v.1, n.2, jan./jun. 2003.

CARZOLA, G.; FARHI, A. Football: exigences physiques et physiologiques actuelles. Revue EPS: éducation physique et sport, n. 273, p.60-66, 1998.

DAVIS, J. A.; BREWER, J.; ATKIN, D. Pre-season physiological characteristics of english first and second division soccer players. Journal of Sports Sciences, v.10, p. 541-547, 1992.

DENADAI, S. B. Índices fisiológicos de avaliação aeróbia: conceitos e aplicações. Rib.Preto: B.S.D.1999.

DUARTE, M. F. S.; DUARTE, C. R. Validade do teste aeróbio de corrida de vai-evem de 20 metros. Revista Brasileira Ciência e Movimento. Brasília: v. 9, n. 3, p. 0714. jul. 2001.

DUFOUR, W. Las técnicas de observacion del comportamiento motor. Fútbol: La observacion tratada mediante ordenador. Stadium, p. 8 -16, 1989.

EKBLOM, B. Applied physiologly of soccer. Sport Medicine, v. 3, p. 50 - 60. 1986.

EKBLOM, B. A field test for soccer players. Science and Football, v. 1, p. $13-15$, 1989.

FAULKNER, J. A. Physiology of swimming and diving. In: FALLS, H. Exercise Physiology. Baltimore: Academic Press, 1968.

FERNANDES, J. L. Futebol: ciencia, arte ou...Sorte! treinamento para profissionais: alto rendimento: preparação física, técnica, tática e avaliação. São Paulo: E. P. U. 1994. FERNANDES FILHO, J. A prática da avaliação fisica. Rio de Janeiro; Ed. Shape, 1999.

FRISSELLI, A.; MANTOVANI, M. Futebol: teoria e prática. São Paulo: Phorte, 1999. 
GODIK, M. A. Futebol: preparação dos futebolistas de alto nível. Rio de Janeiro: Grupo Palestra, 1996.

GODIK, M. A.; POPOV, A. V. La preparación del futbolista. 2 ed. Barcelona: Paidotribo, 1990

GOLOMAZOV, S.; SHIRVA, B. Futebol: preparação física. Londrina: Lazer Sport, 1997.

GREEN, S. Antropometric and physiological characteristicas of South Australian soccer players. Australian Journal of Science and Medicine in Sport, v. 4, n. 1, p. 3-7, 1992.

KACANI, L. La carga física en los entrenamientos y en los partidos de fútbol. Entrenador Español, v. 7, p. 53 - 59, 1981.

LÉGER, L.; GADOURY, C. Validity of the $20 \mathrm{~m}$ shuttle run test with I min stages to predict VO2 max. in adults. Canadian Journal of Spor. Science, v. 14, n. 1, p. 21-26, 1989.

LÉGER, L. A.; LAMBERT, J. Maximal multistage $20 \mathrm{~m}$ shuttles run tes to predict VO2max. European Journal of Applied Physiology, v. 49, p 1-5, 1982.

MATHEWS, D. K. Medidas e avaliação em educação física. Rio de Janeiro: Interamericana, 1980.

MOUTINHO, C. A. As ciências do desporto e a prática desportiva. Atlas, p. $265-$ $275,1981$.

OLIVEIRA, P. R. Treinamento de Resistência nos Esportes. In: CONGRESSO INTERNACIONAL DE ATIVIDADE FÍSICA. São Paulo, 1997. Anais... São Paulo: F.M.U., 1997.

OLIVEIRA, P. R.; AMORIM, C. A . N.; GOULART, L. F., Estudo do esforço físico no futebol junior. Revista Paranaense de Educação Física, v. 1, n.2, p. 49-58, 2000.

PUGGINA, H. et al. Dinâmica da alteração de diferentes capacidades no ciclo anual. Revista Treino Desportivo. (no prelo).

REILLY, T.; THOMAS, V. A motion of work-rate in different positional roles in professional, football match-play. Journal of Human Movement Studies, v. 2, p. 87-97, 1976. 
REILLY, T.; BANGSBO, J.; FRANKS, A. Antropometric and physiological predispositions for elite soccer. Journal of Sports Sciences, v. 18, p. 669-683, 2000.

REILLY, T. et al. A multidisciplinary approach to talent indification in soccer. Journal of Sports Sciences, v. 18, p. 695-702, 2000.

SARTORI, C. R. Estudo das variáveis antropométricas e do desempenho anaeróbioco teste de wingate de atletas de futebol de campo por posição tática. 2000. Monografia (Trabalho de Conclusão de Curso) - Faculdade de Educação Física, Universidade Estadual de Campinas, Campinas, 2000.

SANTOS, J. A. R. Estudo comparativo, fisiológico, antropométrico e motor entre futebolistas de diferente nível competitivo. Revista Paulista de Educação Física, São Paulo, v. 13, n.2, p. 146-159, jul./dez. 1999.

SILVA, M. Caracterização do esforço em modalidades desportivas mensuráveis e não mensuráveis. Treino Desportivo, v. 10, p. 36-46, 1988.

SILVA, P. R. S., ROMANO, A. VISCONTI, A. M. Avaliação funcional multivariada e m jogadores de futebol profissional: uma metanálise. Revista Brasileira Medicina Esporte, v. 4, n. 6, p. 182-196, nov./dez. 1998.

SILVA, P. R. S. et al. Os limites do rendimento físico: considerações fisiometabólicas. Âmbito: medicina desportiva, v. 3, n. 34, p. 13-16, ago. 1997.

SILVA, P. R. S. et al. Efeito do treinamento físico específico nas respostas cardiorrespiratórias e metabólicas em repouso e no exercício máximo em jogadores de futebol profissional. Revista Brasileira de Medicina do Esporte, v. 3, n. 4, p. 101-107, out./dez. 1997.

Perfil de limiares ventilatórios durante o exercício de pico verificado em jogadoras de futebol. Revista Acta Fisiátrica, v. 5, n.2, ago. 1998.

SILVA, P. R. S. et al. A importância do limiar anaeróbio e do consumo máximo de oxigênio em jogadores de futebol. Ambito: medicina desportiva, v. 4, n. 41, p. 15-24, mar., 1998.

SILVA, P. R. S., ROXO, C. D. M. N., VISCONTI, A. M., et al. Índices de aptidão funcional em jogadores de futebol da Seleção Nacional da Jamaica. Revista Brasileira

Medicina Esporte, v.5, n. 3, maio/junho, 1999. 
SILVA, S. G. et al. Association between anthropometric variables with anaerobic power and capacity in brasilian juvenile and junior soccer players. Medicine and Science in Sports and Exercise, v. 32, supl. 5, S181, 2000.

SMAROS, G. Energy usagy during football match. In: CONGRESS ON SPORTS MEDICINE APPLIED TO FOOTBALL, 1. Roma, 1980. Proceedings... Roma, p. 795801, 1980. v. 2.

VERKOSHANSKY, Y. V. Entrenamiento deportivo: planificación y programación. Barcelona: Martínez Roca. 1990.

VERKHOSANSKY, Y. V. Problemas atuais da metodologia do treino desportivo. Revista Treinamento Desportivo, v. 1, n. 1, p. 33-45, 1996.

WEINECK, J. Manual de treinamento esportivo. 2 ed. São Paulo: Manole, 1986. Treinamento ideal . 9. ed. São Paulo: Manole, 1999.

Futebol total: o treinamento físico no futebol. Guarulhos: Phorte, 2000.

WINTERS, R.T. et al. Match analysis of Australian professional soccer players. Journal of Human Movement Studies, p. 159-176, 1982. 